\title{
Olfm4 deletion enhances defense against Staphylococcus aureus in chronic granulomatous disease
}

\author{
Wenli Liu, ${ }^{1}$ Ming Yan, ${ }^{2}$ Janyce A. Sugui, ${ }^{3}$ Hongzhen Li, ${ }^{1}$ Chengfu Xu, ${ }^{2}$ Jungsoo Joo, ${ }^{2}$ \\ Kyung J. Kwon-Chung, ${ }^{3}$ William G. Coleman, ${ }^{2}$ and Griffin P. Rodgers ${ }^{1}$ \\ ${ }^{1}$ Molecular and Clinical Hematology Branch, National Heart, Lung, and Blood Institute, ${ }^{2}$ Laboratory of Biochemistry and Genetics, \\ National Institute of Diabetes and Digestive and Kidney Diseases, and ${ }^{3}$ Laboratory of Clinical Infectious Diseases, \\ National Institute of Allergy and Infectious Diseases, NIH, Bethesda, Maryland, USA.
}

\begin{abstract}
Chronic granulomatous disease (CGD) patients have recurrent life-threatening bacterial and fungal infections. Olfactomedin 4(OLFM4) is a neutrophil granule protein that negatively regulates host defense against bacterial infection. The goal of this study was to evaluate the impact of Olfm 4 deletion on host defense against Staphylococcus aureus and Aspergillus fumigatus in a murine X-linked gp91phox-deficiency CGD model. We found that intracellular killing and in vivo clearance of $S$. aureus, as well as resistance to $S$. aureus sepsis, were significantly increased in $g$ p91phox and Olfm 4 double-deficient mice compared with CGD mice. The activities of cathepsin $C$ and its downstream proteases (neutrophil elastase and cathepsin G) and serum levels of IL-1 $\beta$, IL-6, IL-12p40, CXCL2, G-CSF, and GM-CSF in Olfm4-deficient as well as gp91phox and Olfm 4 double-deficient mice were significantly higher than those in WT and CGD mice after challenge with $S$. aureus. We did not observe enhanced defense against $A$. fumigatus in $O$ lfm4-deficient mice using a lung infection model. These results show that $\mathrm{Olfm} 4$ deletion can successfully enhance immune defense against $S$. aureus, but not $A$. fumigatus, in CGD mice. These data suggest that OLFM4 may be an important target in CGD patients for the augmentation of host defense against bacterial infection.
\end{abstract}

\section{Introduction}

Chronic granulomatous disease (CGD) comprises a group of recessive inherited disorders characterized by recurrent life-threatening bacterial and fungal infections with granuloma formation (1). The disease results from mutations in any one of four subunits of the respiratory burst oxidase (NADPH oxidase) found in phagocytic leukocytes. Two-thirds of CGD cases are due to X-linked gp91phox deficiency (2). NADPH oxidase generates superoxide, the precursor to hydrogen peroxide and other reactive oxygen derivatives with microbicidal activity (3). The relative resistance of microbes to nonoxidative killing mechanisms, such as defensins, bactericidal/permeability-increasing factor, lactoferrin (4), and other lysosomal proteases, may also play a role in the distinctive pattern of microbial infection seen in CGD (5). The delivery of superoxide by the NADPH oxidase complex into the phagosome is also thought to indirectly activate several classes of proteases by increasing $\mathrm{K}^{+}$influx and $\mathrm{pH}(6)$.

Olfactomedin 4 (OLFM4) is a member of the olfactomedin domain-containing glycoprotein family whose expression is known to be regulated by G-CSF, PU.1, and NF-KB (7). OLFM4 is stored in the granules of mature neutrophils (8). Recent function studies revealed that OLFM4 is an important modifier of neutrophil bacterial killing and host innate immunity against Gram-positive and Gram-negative bacteria, including Staphylococcus aureus, Escherichia coli (8), and Helicobacter pylori (9). These features are mediated through its restriction on neutrophil cathepsin C-mediated protease activities (8) and nucleotide-binding oligomerization domain-mediated (NOD-mediated) NF-кB acti-

Authorship note: Wenli Liu and Ming Yan contributed equally to this work. Conflict of interest: The authors have declared that no conflict of interest exists. Citation for this article: J Clin Invest. 2013;123(9):3751-3755. doi:10.1172/JCI68453. vation (9). NADPH oxidase-mediated bacterial killing is totally abolished in CGD patients. Here, we hypothesize that deletion of Olfm4 in CGD neutrophils may restore microbe-killing activities through nonoxidative mechanisms.

\section{Results and Discussion}

In normal neutrophils, microorganisms are killed by the combined action of oxygen-dependent and oxygen-independent mechanisms. CGD neutrophils possess only oxygen-independent mechanisms, and killing mainly depends on the microbicidal effect of the neutrophil granule proteins (5). In this study, we first investigated whether deletion of Olfm4, a negative modulator of host defense against bacterial infection, could boost X-linked CGD mouse immunity against $S$. aureus, a commonly encountered pathogen in CGD patients. X-CGD mice have a null allele for gp91phox and are similar to patients with CGD in that they manifest impaired resistance to infections with $S$. aureus and Aspergillus fumigatus (10). Nitroblue tetrazolium (NBT) reduction assays (Figure 1A) and superoxide burst assays (Figure 1B) confirmed the lack of NADPH oxidase activity in gp91phox-deficient mice.

We first crossed gp91phox-deficient mice with Olfm4-deficient mice to create gp91phox and Olfm 4 double-deficient mice. These double-deficient mice demonstrated normal development and growth without symptoms of colitis or skin disease. We then analyzed neutrophils derived from the bone marrow of mice with different genotypes for their intracellular killing of two strains of S. aureus: Rosenbach and USA300/LAC, the latter of which is a methicillin-resistant $S$. aureus (MRSA). In both strains, the neutrophils from gp91phox ${ }^{+/+}$Olfm $4^{-/-}$(Olfm4-deficient) mice demonstrated increased capability to kill intracellular $S$. aureus, while neutrophils from gp91phox-/-Olfm $4^{+/+}$(CGD) mice had decreased bacterial-kill- 
A
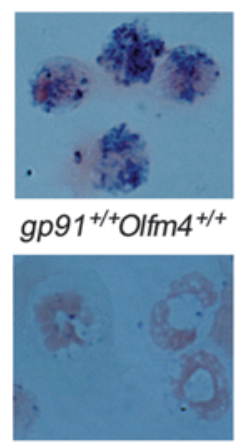

gp $91^{1 /-O l f m} 4^{+/-}$
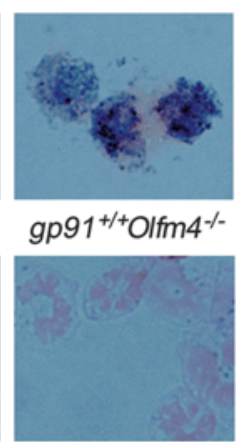

gp $91 \%$ Olfm 4 -

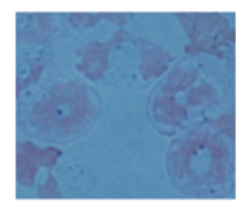

gp91\% Olfm $4^{+/+}$

B

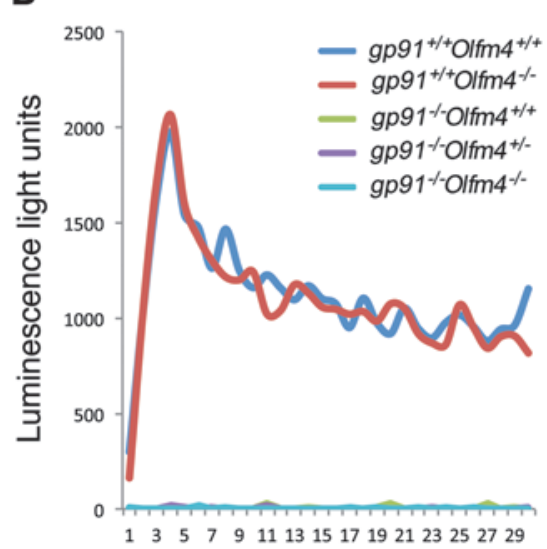

c
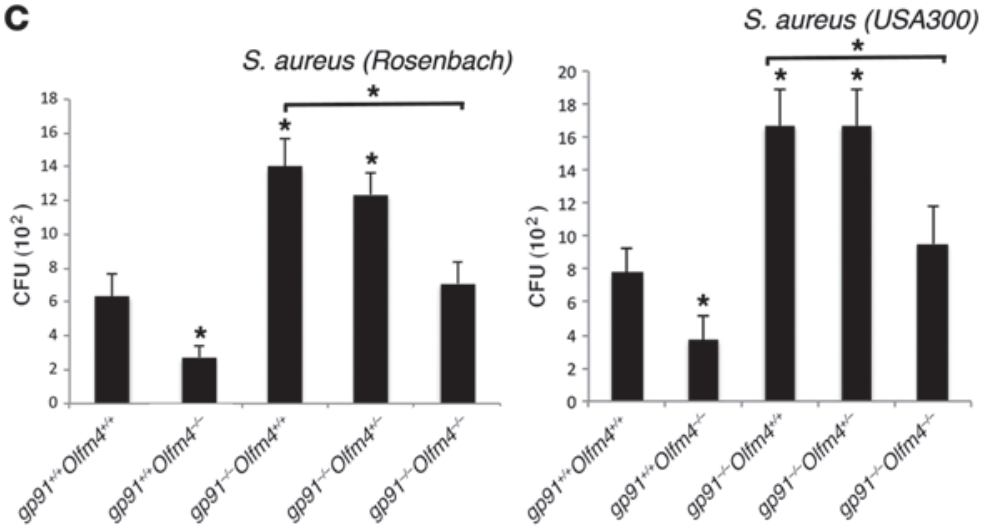

S. aureus (USA300)

D

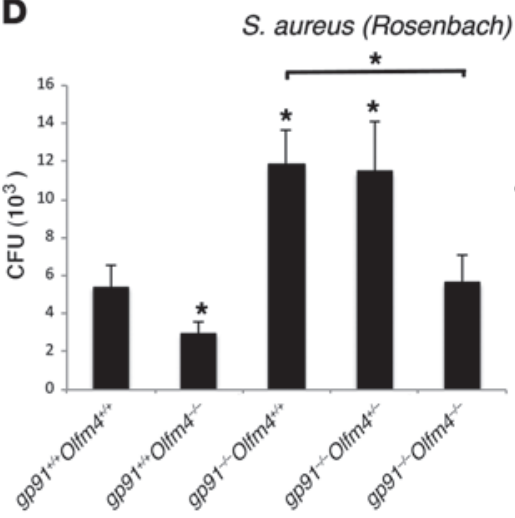

*

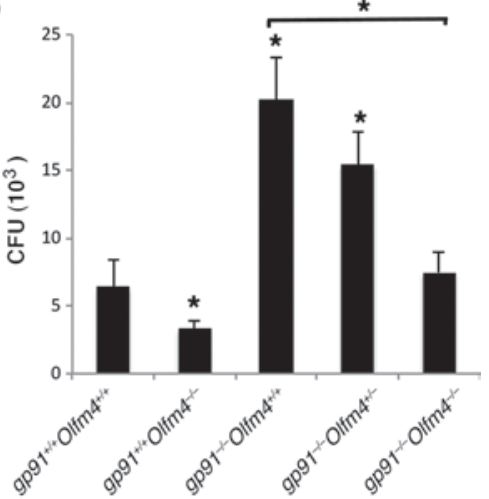

Figure 1

Intracellular killing and in vivo peritoneal clearance of S. aureus in Olfm4- and gp91phox-deficient mice. (A) NBT assays and (B) superoxide burst assays were performed to confirm the loss of NAPDH oxidase activity in different genotypes of mice. Original magnification, $\times 200$ in NBT assays. (C) Neutrophils derived from the bone marrow of Olfm4- and gp91phox-deficient mice were incubated with preopsonized S. aureus (Rosenbach or USA300). The number of viable bacteria (CFU) after the cells were treated with gentamicin $(50 \mu \mathrm{g} / \mathrm{ml})$ was determined on plates. Data are expressed as the mean \pm SD $(n=5)$. (D) Mice were challenged i.p. with S. aureus (Rosenbach or USA300). After 6 hours, the peritoneal cavity was lavaged, and the number of viable bacteria (CFU) was determined on plates. Data are expressed as the mean \pm SD $(n=5)$. ${ }^{\star} P<0.05$ when compared with WT (gp91phox $\left.{ }^{+/+O} \mathrm{Olfm} 4^{+/+}\right)$mice or as indicated.

ing ability compared with those from $g$ p91phox ${ }^{+/+} \mathrm{Olfm} 4^{+/+}$(WT) mice (Figure 1C). The bacterial-killing abilities in $g p 91$ phox ${ }^{-1-}$ Olfm $4^{-/-}$(double-deficient) neutrophils were restored to the levels of those from WT mice (Figure 1C). Neutrophils from $g p 91$ phox ${ }^{-/-}$ Olfm $4^{+/-}$and gp91phox-/-Olfm $4^{+/+}$(CGD) mice demonstrated a similar level of bacterial killing (Figure $1 \mathrm{C}$ ). We then performed in vivo peritoneal $S$. aureus clearance assays for the two strains among different genotypes of mice. Similarly, Olfm4-/- (Olfm4-deficient) mice had increased in vivo peritoneal $S$. aureus clearance, and gp91phox ${ }^{-/-} \mathrm{Olfm}^{+/+}$(CGD) mice had decreased bacterial-clearance ability. In addition, double-deficient mice had enhanced in vivo bacterial clearance abilities compared with CGD mice and demonstrated a similar level of bacterial clearance ability to that of WT mice (Figure 1D). These results indicate that a homozygous deletion of the Olfm 4 gene in CGD mice could enhance CGD mouse $S$. aureus killing and in vivo clearance.

Next, we investigated whether deletion of Olfm 4 in CGD mice could increase the host defense against systemic infections of $S$. aureus and A. fumigatus, the latter of which is another common pathogen in CGD patients. Mice of various genotypes were infected with S. aureus of Rosenbach or USA300 by i.p. injection, and their survival was monitored. All CGD mice and $g p^{9} 91$ phox ${ }^{-/-O l f m} 4^{+/-}$mice died within 2 to 5 days (Figure 2A). Most WT mice died gradually during a 2-week observation period. In contrast, almost all the Olfm4-deficient and gp91phox and Olfm4 double-deficient mice survived. Consistent with our earlier observations, these results suggest that a homozygous, but not a heterozygous, deletion of Olfm 4 could increase host system defense against $S$. aureus infection and prevent $S$. aureus sepsis-induced mortality in CGD mice.

Pulmonary aspergillus infection is the most common microbial cause of death in CGD patients, and NADPH oxidase-deficient mice are also susceptible to experimental aspergillus challenge (11). Therefore, we wanted to determine whether OLFM4 is involved in the mouse host defense against A. fumigatus using a lung infection model. Following oropharyngeal challenge with a moderate inoculum $\left(5 \times 10^{5}\right.$ conidia per mouse), CGD, double-deficient, and gp91phox-/-Olfm $4^{+/-}$mice all died within 9 days, whereas WT and Olfm4-deficient mice all survived (Figure 2B). 

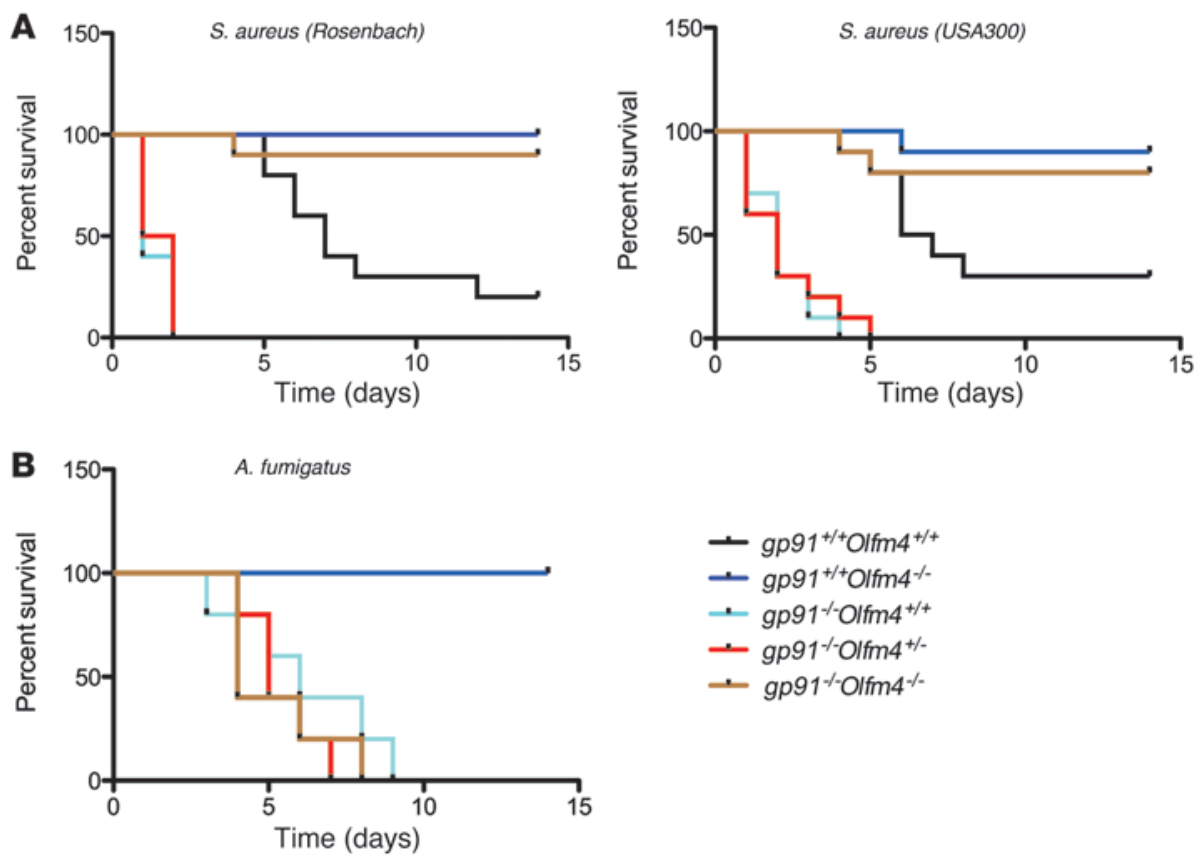

\section{Figure 2}

Susceptibility of Olfm4- and gp91phoxdeficient mice to $S$. aureus or A. fumigatus infection. (A) Survival probability plots (Kaplan-Meier) of experimental groups infected i.p. with S. aureus (Rosenbach or USA300) $\left(2 \times 10^{8}\right.$ CFU per mouse; $\left.n=10\right)$. Survival was monitored every 6 hours. (B) Survival probability plots (Kaplan-Meier) of experimental groups infected with oropharyngeal aspiration of $A$. fumigatus $\left(5 \times 10^{5}\right.$ CFU per mouse; $\left.n=5\right)$. Survival was monitored twice a day.
These results showed that Olfm 4 deletion did not prevent pulmonary A. fumigatus infection-induced death in CGD mice. We also evaluated host immune response in $\mathrm{Olfm} 4^{-/-}$mice compared with WT mice 24 hours after a high $A$. fumigatus inoculum $\left(1 \times 10^{7}\right.$ conidia per mouse). Bronchoalveolar fluid leukocytosis was not significantly different between the two genotypes (Supplemental Figure 1A; supplemental material available online with this article; doi:10.1172/JCI68453DS1). No significant difference between the two genotypes was observed in the quantitative fungal burden in the lungs (Supplemental Figure 1B) or in the Grocott-Gomori methenamine-silver staining in lung sections (Supplemental Figure 1C). Lung histology showed a similar level of inflammation characterized by peribronchial and perivascular inflammatory cell infiltration, including neutrophils and mononuclear cells, in Olfm $4^{-/-}$and WT mice (Supplemental Figure 1D). Taken together, these results suggest that OLFM4 may not play a role in the mouse host defense against pulmonary aspergillosis.

Neutrophils from gp91phox-deficient mice or CGD patients have impaired fungicidal activity, and the generation of ROS by neutrophils is considered to be the major antifungal mechanism lacking in CGD (12). Although evidence suggests that neutrophils can mediate antifungal activities via nonoxidative mechanisms, including defensins (13), lactoferrin (4), and pentraxins (14), neutrophils from CGD patients do not cause substantial damage to hyphae in vitro (15), suggesting that cellular nonoxidative mechanisms alone are insufficient to kill hyphae under physiologic conditions. It has been reported that $A$. fumigatus was markedly resistant to neutrophil granule extract (5). Neutrophils use oxygen-dependent mechanisms to attack hyphae germinating from conidia that escape alveolar macrophage surveillance (16). A recent study demonstrated that neutrophil proteases and cathepsin $\mathrm{C}$, which are important for bacterial innate immunity $(17,18)$, do not play a role in mouse host defense against pulmonary aspergillosis (11). Because OLFM4 regulates bacterial killing largely through modulation of cathepsin C and neutrophil proteases in the granules (8), this may explain why
Olfm4 deletion did not alter the mice's defense against A. fumigatus, but led to enhanced host defense against $S$. aureus. These results support the conclusion that NADPH oxidase and neutrophil proteases have distinct antibacterial and antifungal functions.

To explore the mechanism through which Olfm 4 deletion enhanced the bactericidal activities of CGD neutrophils, we analyzed the activities of cathepsin $C$ and its downstream proteases (neutrophil elastase and cathepsin G) in the neutrophils from mice after $S$. aureus challenge. Cathepsin C activity in the neutrophils of Olfm4-deficient mice and double-deficient mice was significantly higher than that in WT mouse neutrophils, while cathepsin C activity in CGD ( $g$ p91phox $x^{-/-} \mathrm{Olfm}_{4++}^{++}$) and gp91phox $x^{-/-O} \mathrm{lfm}_{4}^{+/-}$ mice was similar to that in WT mice (Figure 3A). Accordingly, the neutrophil elastase (Figure 3B) and cathepsin G (Figure 3C) activities in Olfm4-deficient and double-deficient mice were also substantially higher than those in WT mice as well as in CGD and gp91phox ${ }^{-/-O l f m} 4^{+/-}$mice. These results suggest that the increase in serine protease activities observed with Olfm 4 deletion is NADPH independent. The compromised neutrophil bacterial killing and host innate immunity against $S$. aureus in CGD mice due to oxidative mechanism deficiency could be successfully restored by the enhancement of serine protease activities in neutrophils subsequent to $\mathrm{Olfm} 4$ deletion. Serine proteases are stored in granules in their active form until they are released following neutrophil exposure to inflammatory stimuli. Once released, neutrophil serine proteases are potentially fully active and have broad biological effects, including intracellular microbial killing and modulation of inflammatory cell recruitment (19).

A previous study showed that OLFM4 binds NOD1 and NOD2 and inhibits NOD-mediated NF-кB signaling (9). Here, we analyzed cytokine and chemokine levels in the serum of mice following challenge with $S$. aureus. The levels of IL-1 $\beta$, IL-6, IL-12p40, CXCL2, G-CSF, and GM-CSF were significantly higher in the Olfm4-deficient mice as well as in the gp91phox and Olfm 4 double-deficient mice than the levels in WT and CGD mice (Figure 3D). All of these cytokines 
A

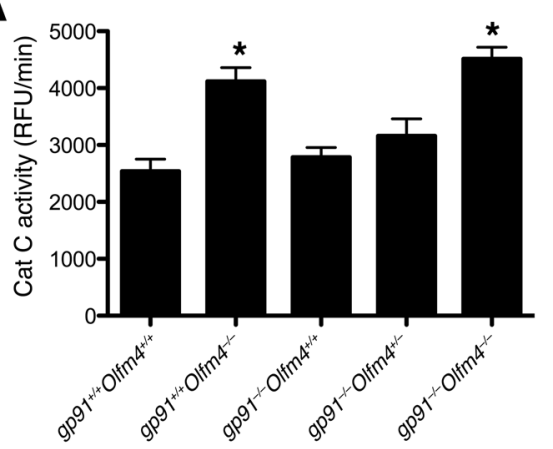

D
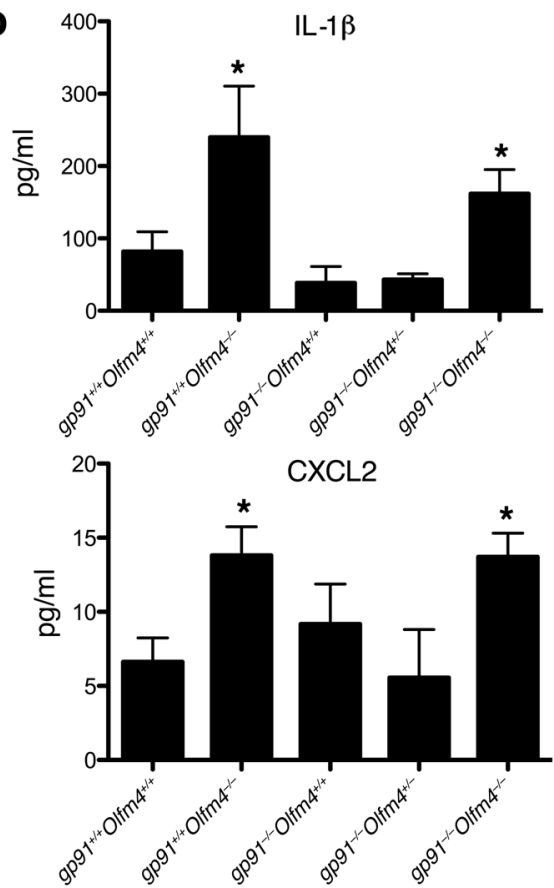

B
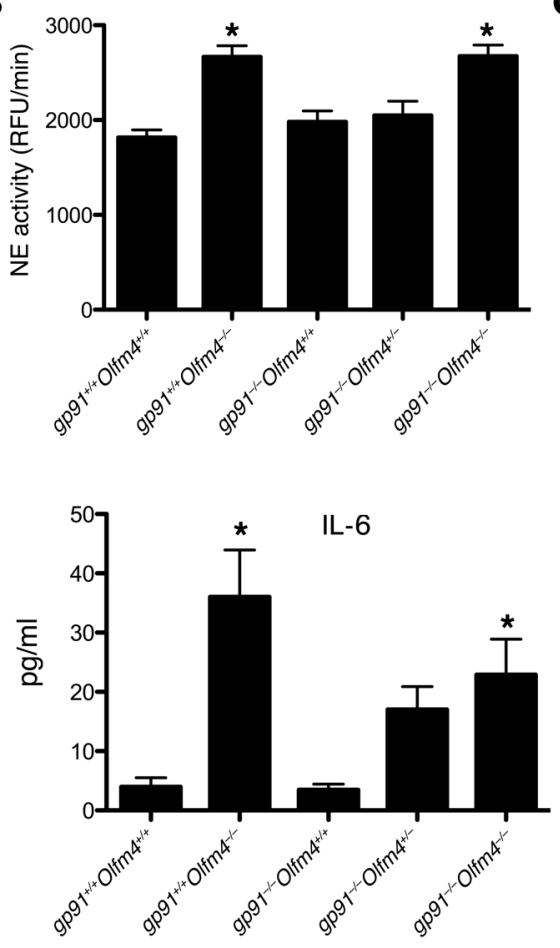

G-CSF

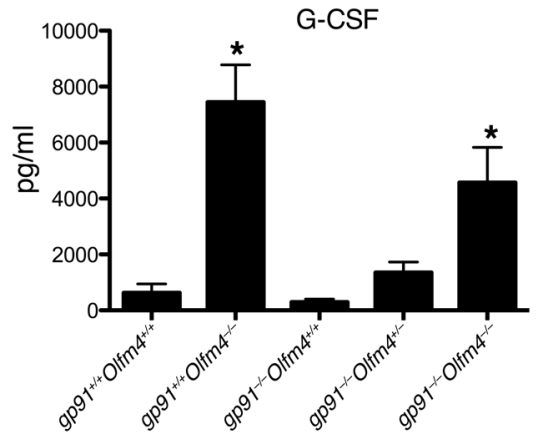

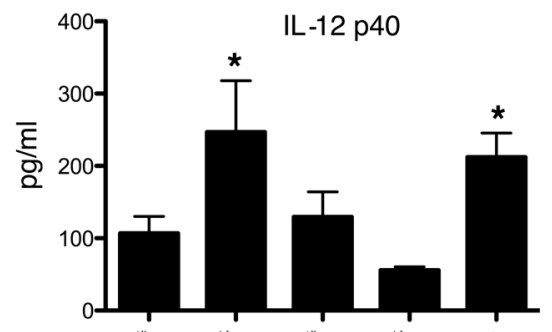

C
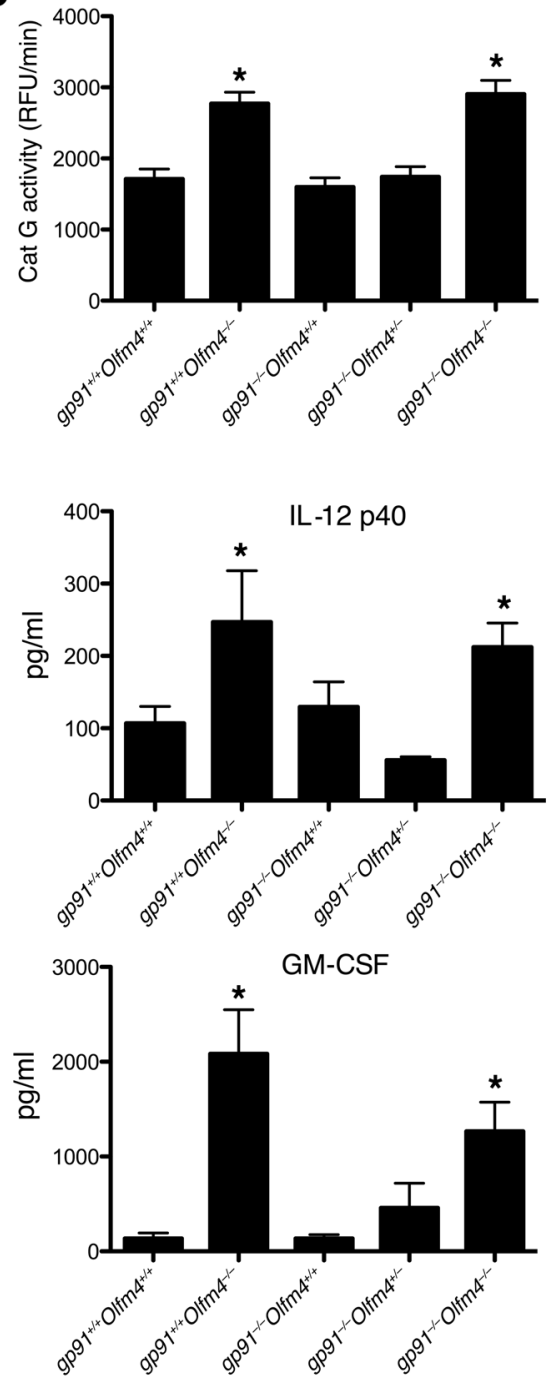

Figure 3

Cathepsin C and serine protease activities and cytokine/chemokine serum levels in Olfm4- and gp91phox-deficient mice. (A-C) Neutrophils $\left(5 \times 10^{6}\right)$ derived from the bone marrow of mice with different genotypes 6 hours after i.p. infection with S. aureus $\left(5 \times 10^{7} \mathrm{CFU}\right.$ per mouse) were lysed, and an equal amount of lysate was used for assays of cathepsin $\mathrm{C}(\mathrm{Cat} \mathrm{C})(\mathbf{A})$, neutrophil elastase $(\mathbf{B})$, and cathepsin $\mathrm{G}(\mathrm{Cat} \mathrm{G})(\mathbf{C})$ activity using the corresponding AMC-labeled substrate. ${ }^{*} P<0.05$ when compared with WT (gp91 phox $\left.{ }^{+/+} \mathrm{Olfm} 4^{+/+}\right)$mice. Data are expressed as the mean $\pm \mathrm{SD}(n=5)$. RFU, relative fluorescence unit. (D) Cytokine and chemokine levels in the serum of mice with different genotypes 6 hours after i.p. infection with S. aureus $\left(5 \times 10^{7} \mathrm{CFU}\right.$ per mouse) were determined by high-throughput immunoassay. Data are expressed as the mean \pm SD for each experimental group $(n=5)$. ${ }^{*} P<0.05$ versus WT (gp91phox ${ }^{+/+O}$ lfm $4^{+/+}$) mice.

and chemokines are known target genes of the NF-KB pathway. We consistently found that NF-KB activity in the neutrophils from Olfm 4-deficient as well as gp91phox and Olfm 4 double-deficient mice was significantly higher than that in WT and CGD mice (Supplemental Figure 2). The enhanced cytokine levels in Olfm4-deficient mice could be caused by the upregulated NF-KB signaling as well as by increased serine protease activities. Neutrophil serine proteases might not only regulate the activity of chemokines and cytokines by proteolysis, but might also modulate their release through cellular activation (19). NOD2 is critical for innate recognition and antibacterial defense against $S$. aureus $(20,21)$. Therefore, the enhanced NOD-mediated defense pathways caused by $\mathrm{Olfm} 4$ deletion may also contribute to the enhanced host defense against $S$. aureus in CGD.
NOD signaling works synergistically with TLR signaling to recognize S. aureus infection and induce inflammatory responses (22). The TLR2-MyD88 pathway has been recognized to play important roles in mouse innate immunity against $S$. aureus infection (23). To determine whether the effect of Olfm 4 deletion on S. aureus immune defense is mediated through the TLR-MyD88 pathway, we investigated the host defense of MyD88 and Olfm 4 double-deficient mice against $S$. aureus systemic infection. While the MyD88-deficient mice were highly susceptible to $S$. aureus infection, additional Olfm 4 elimination remarkably improved the survival of $M y D 88$ deficient mice and enhanced the serum levels of some NF-kB-targeted pro-inflammatory cytokines and chemokines (Supplemental Figure 3). These results suggest that enhanced mouse innate 
immunity due to Olfm4 deletion is TLR-MyD88 signal independent and that NOD-mediated NF- $\mathrm{KB}$ may play an important role. The mechanism of OLFM4 regulation of host immune defense against S. aureus is summarized in Supplemental Figure 4.

Taken together, we found that deletion of Olfm 4 , a critical negative regulator of neutrophil protease activities and the NODmediated pathway, could enhance the immune defense against $S$. aureus infection in CGD mice. This finding provides a rationale for enhancing CGD patient defense against bacterial infections potentially through the modulation of OLFM4 levels.

\section{Methods}

Further information can be found in Supplemental Methods.

Mice, bacteria, and fungi. X-linked gp91phox-deficient mice on a C57BL/6 background were purchased from The Jackson Laboratory. Olfm4deficient mice backcrossed six generations onto a C57BL/ 6 background were described previously (8). Olfm 4 and gp91phox-double-deficient mice were derived from crossing Olfm4-deficient and gp91phox-deficient mice. Animals were kept in a specific pathogen-free facility at the NIH. All experiments were conducted with 8- to 10-week-old male animals. S. aureus (Rosenbach) ATCC 10390 was purchased from ATCC, and S. aureus (USA300/LAC) was provided by Frank R. DeLeo of the NIH. Bacteria were grown in tryptic soy broth or on tryptic soy agar plates (Teknova). A. fumigatus strain B-5233, a pulmonary clinical isolate from a leukemic patient, was maintained on Aspergillus minimal medium (4). Conidia were harvested from 1-week-old cultures with PBS and quantitated on a hematocytometer.

Intracellular bacterial-killing assay. Purification of bone marrow-derived mouse neutrophils and the bacterial-killing assay were performed as described previously (8).

Protease activity assays. The assays were performed as previously described (8) with minor modifications. Cathepsin C activity was assayed in $25 \mathrm{mM}$ 2-ethanesulfonic acid (MES), pH 6.0, $50 \mathrm{mM} \mathrm{NaCl}, 5 \mathrm{mM}$ DTT, and $0.1 \%$ polyethylene glycol (PEG) 3350 using Gly-Arg-AMC (Bachem) at $10 \mu \mathrm{M}$. Neutrophil elastase (NE) activity was assayed in $100 \mathrm{mM}$ Tris-HCl, pH 7.5, and $50 \mathrm{mM} \mathrm{NaCl}$ using Ala-Ala-Pro-Val-AMC (Bachem) as a substrate (100 $\mu \mathrm{M})$. Cathepsin G activity was assayed in $100 \mathrm{mM}$ Tris- $\mathrm{HCl}, \mathrm{pH} 7.5$, and 50 $\mathrm{mM} \mathrm{NaCl}$ using Ala-Ala-Pro-Phe-AMC (Bachem) as a substrate $(100 \mu \mathrm{M})$. Reaction progress was monitored continuously with product (AMC) on a FLUOstar OPTIMA Fluorimeter (BMG LABTECH) with 380-nm excitation and 460-nm emission wavelength filters.

Statistics. Kaplan-Meier curves were generated with GraphPad Prism 5.0 software (GraphPad) and analyzed using the log-rank method. The Mann-Whitney $U$ test was used to compare the quantitation of CFU, fungal burden, and cytokine levels. A $P$ value of less than 0.05 was considered statistically significant.

Study approval. All animal procedures were conducted according to protocols approved by the National Heart, Lung, and Blood Institute and the National Institute of Allergy and Infectious Diseases Animal Care and Use committees.

\section{Acknowledgments}

We thank John I. Gallin and Kol A. Zarember of the Clinical Center, NIH, for their helpful discussions and careful review of the manuscript. We thank Frank R. DeLeo of the NIH for providing S. aureus (USA300/LAC).

Received for publication December 19, 2012, and accepted in revised form May 30, 2013.

Address correspondence to: Griffin P. Rodgers, Molecular and Clinical Hematology Branch, National Heart, Lung, and Blood Institute, NIH, Bldg. 10, Room 9N119, 9000 Rockville Pike, Bethesda, Maryland 20892, USA. Phone: 301.402.2418; Fax: 301.480.1940; E-mail: gr5n@nih.gov. Or to: William G. Coleman, Laboratory of Biochemistry and Genetics, National Institute of Diabetes and Digestive and Kidney Diseases, NIH, Bldg. 3, Room 5E08, 3 Center Drive, Bethesda, Maryland 20892, USA. Phone: 301.402.1366; Fax: 312.350.2077; E-mail: wcoleman@helix.nih.gov.
1. Malech HL, Nauseef WM. Primary inherited defects in neutrophil function: etiology and treatment. Semin Hematol. 1997;34(4):279-290.

2. Malech HL. Progress in gene therapy for chronic granulomatous disease. JInfect Dis. 1999;179(suppl 2):S318-S325.

3. Hampton MB, Kettle AJ, Winterbourn CC. Inside the neutrophil phagosome: oxidants, myeloperoxidase, and bacterial killing. Blood. 1998;92(9):3007-3017.

4. Zarember KA, Sugui JA, Chang YC, Kwon-Chung KJ, Gallin JI. Human polymorphonuclear leukocytes inhibit Aspergillus fumigatus conidial growth by lactoferrin-mediated iron depletion.J Immunol. 2007;178(10):6367-6373.

5. Odell EW, Segal AW. Killing of pathogens associated with chronic granulomatous disease by the non-oxidative microbicidal mechanisms of human neutrophils. J Med Microbiol. 1991;34(3):129-135.

6. Reeves EP, Lu H, Jacobs HL, et al. Killing activity of neutrophils is mediated through activation of proteases by K ${ }^{+}$flux. Nature. 2002;416(6878):291-297.

7. Tomarev SI, Nakaya N. Olfactomedin domain-containing proteins: possible mechanisms of action and functions in normal development and pathology. Mol Neurobiol. 2009;40(2):122-138.

8. Liu W, Yan M, Liu Y, McLeish KR, Coleman WG $\mathrm{Jr}$, Rodgers GP. Olfactomedin 4 inhibits cathepsin C-mediated protease activities, thereby modulating neutrophil killing of staphylococcus aureus and escherichia coli in mice. I Immunol. 2012;189(5):2460-2467.

9. Liu W, et al. Olfactomedin 4 down-regulates innate immunity against Helicobacter pylori infection. Proc Natl Acad Sci U S A. 2010;107(24):11056-11061.

10. Pollock JD, et al. Mouse model of X-linked chronic granulomatous disease, an inherited defect in phagocyte superoxide production. Nat Genet. 1995;9(2):202-209.

11. Vethanayagam RR, et al. Role of NADPH oxidase versus neutrophil proteases in antimicrobial host defense. PLoS One. 2011;6(12):e28149.

12. Park SJ, Mehrad B. Innate immunity to Aspergillus species. Clin Microbiol Rev. 2009;22(4):535-551.

13. Levitz SM, Selsted ME, Ganz T, Lehrer RI, Diamond RD. In vitro killing of spores and hyphae of Aspergillus fumigatus and Rhizopus oryzae by rabbit neutrophil cationic peptides and bronchoalveolar macrophages. J Infect Dis. 1986;154(3):483-489.

14. Jaillon $S$, et al. The humoral pattern recognition receptor PTX3 is stored in neutrophil granules and localizes in extracellular traps. J Exp Med. 2007;204(4):793-804.

15. Diamond RD, Clark RA. Damage to Aspergillus fumigatus and Rhizopus oryzae hyphae by oxidative and nonoxidative microbicidal products of human neutrophils in vitro. Infect Immun. 1982; 38(2):487-495

16. Schaffner A, Douglas H, Braude A. Selective protec- tion against conidia by mononuclear and against mycelia by polymorphonuclear phagocytes in resistance to Aspergillus. Observations on these two lines of defense in vivo and in vitro with human and mouse phagocytes. J Clin Invest. 1982;69(3):617-631.

17. Belaaouaj A, et al. Mice lacking neutrophil elastase reveal impaired host defense against gram negative bacterial sepsis. Nat Med. 1998;4(5):615-618.

18. Pham CT, Ivanovich JL, Raptis SZ, Zehnbauer B, Ley TJ. Papillon-Lefevre syndrome: correlating the molecular, cellular, and clinical consequences of cathepsin C/dipeptidyl peptidase I deficiency in humans. J Immunol. 2004;173(12):7277-7281.

19. Pham CT. Neutrophil serine proteases: specific regulators of inflammation. Nat Rev Immunol. 2006;6(7):541-550.

20. Hruz P, et al. NOD2 contributes to cutaneous defense against Staphylococcus aureus through alpha-toxin-dependent innate immune activation. Proc Natl Acad Sci U S A. 2009;106(31):12873-12878.

21. Deshmukh HS, et al. Critical role of NOD2 in regulating the immune response to Staphylococcus aureus. Infect Immun. 2009;77(4):1376-1382.

22. Takeda K, Akira S. Toll-like receptors in innate immunity. Int Immunol. 2005;17(1):1-14.

23. Takeuchi O, Hoshino K, Akira S. Cutting edge: TLR2-deficient and MyD88-deficient mice are highly susceptible to Staphylococcus aureus infection. J Immunol. 2000;165(10):5392-5396. 